\title{
Preface to the Best Papers from CLEI 2013 Special Issue
}

\author{
Jose Aguilar ${ }^{1}$, Jaelson Castro ${ }^{2}$, Sergio España ${ }^{3}$, Alexandra La Cruz ${ }^{4}$, María \\ Villapol $^{5}$, Paula Zabala ${ }^{6}$ \\ 'Universidad de Los Andes, Venezuela, aguilar@ula.ve \\ ${ }^{2}$ Universidade Federal de Pernambuco, Brasil, jbc@cin.ufpe.br \\ 3Universidad Politécnica de Valencia, España, sergio.espana@dsic.upv.es \\ ${ }^{4}$ Universidad Simón Bolívar, Venezuela, alexandra.lacruz@gmail.com \\ 5Universidad Central de Venezuela, Venezuela, maria.villapol@ciens.ucv.ve \\ ${ }^{6}$ Universidad de Buenos Aires, Argentina, pzabala@dc.uba.ar
}

This special issue of the CLEI Electronic Journal consists of extended and revised versions of a selection of the best papers presented at the XXXIX Latin American Computing Conference (CLEI 2013), which took place in October 2013 in Naiguatá, Venezuela. The editors for this special issue were the chairs of the symposia from CLEI 2013 where these papers have been selected, plus the general chair of the program committee, which have participated in the selection and revision process.

CLEI is the annual most important conference in Latin America in the computer science discipline. These last years there have been a great effort to provide high quality at the conference: the acceptance rate is around 30 percent, at least 3 peer reviews of the articles, among other things. In addition, in recent editions has tried to publish the best papers in very prestigious journals. Particularly, the program committee of the conference selected a number of papers that were considered especially meritorious, with a theoretical content, to be published in Elsevier's ENTCS (without any change from the conference papers), while the more applied meritorious papers were invited to submitted an extended version to be included in this special issue of CLEI Electronic Journal.

In total, 17 papers of applied content were determined to be meritorious and were invited to be considered for the special issue. These new paper versions were an extended version of the conference paper and were written in English. After an additional refereeing process, the final outcome was that ten papers were selected; contained in this special issue.

The selection has been very hard. More than four hundred papers were submitted for evaluation in the different symposiums, and from the accepted papers, only 17 were selected for this special number. Two aspects have been considered during this selection: the high score in the evaluation process and the contribution of the paper. The papers have been selected from five of the seven CLEI 2013 symposia: from Software Engineering, from Informatics and Society, from Operations Research and Artificial Intelligence, from Infrastructure, Hardware and Software, and from Computer Graphics, Virtual Reality, and Image Processing. A short abstract of these papers is presented below. 
From the Software Engineering Symposium three papers were selected. The first one, "Unified Process for Domain Analysis integrating Quality, Aspects and Goals", by F. Losavio, A. Matteo, and I. Pacilli, proposes a Unified Process for Domain Analysis (UPDA), based on Aspect and Goal orientations to deal with non-functional requirements (NFR), specified by quality standards to enhance communication. UPDA integrates techniques that are separately used: -the Chung and others extended process of Losavio and others, based on the NFR Framework with treatment of crosscutting concerns, and the ISO/IEC 25010 quality standard to specify NFR. The second paper, entitled "Business Process Verification: The Application of Model Checking and Timed Automata", by L. Mendoza, presents how model checking verification technique for software and timed automata formal language is integrated within a formal verification approach to check Business Processes. The last one, titled "A Business Process Model for IT Management Based on Enterprise Architecture", by J. Montilva, J. Barrios and I. Besembel, presents a business process model for the IT Management that can be used in medium and large organizations as a framework for modelling and analysing their IT management processes. The main difference between the described model and others in the literature is that their model places Enterprise Architecture concept at the centre of the organization of IT Management activities.

From the Informatics and Society Symposium two papers were selected. The first one, titled "An experience of technology transfer success of software for children with disabilities", by J. Jadán, presents the development of AINIDIU (Intelligent agent for visually impaired children), which is a computer-based technology to facilitate the interaction between visually impaired children and computers. Additionally, the paper describes the experience of technology transfer of software for visually impaired children in Ecuador. The second one, titled "Free and Open Source Software in Costa Rican Local Governments", by f. Mata and A. Quesada, presents and discusses the results from an electronic survey concerning the use of free and open source software (FOSS) in the local governments in Costa Rica. Comparison of the results from this survey with those obtained from a previous one, shows that the Costa Rican local governments are increasingly using FOSS.

The Operations Research and Artificial Intelligence Symposium has selected two papers. The paper "Evolution of cooperation in evolutionary games for Sanitation Boards", by R. Botta, G. Blanco and C. Schaerer, analyzes several models of a group of individuals that come together to produce a good or provide a service, based on the evolutionary game theory and public good games. They propose that evolutionary game theory can be a useful tool for policy-makers in order to improve cooperation and discourage defection. The second one, entitled "Collective Learning inMulti-Agent Systems Based on Cultural Algorithms", by J. Terán, J. Aguilar, M. Cerrada, presents a learning model for coordination schemes in Multi-Agent Systems based on Cultural Algorithms. In this model, the individuals are the different conversations that may occur in any multi-agent systems, and the coordination scheme learned is at the level of the way to perform the communication protocols into the conversation. They develop a computational tool based on this learning model, called CLEMAS. 
The Infrastructure, Hardware and Software Symposium chose two papers. The paper "An explicit parallelism study based on thread-level speculation", by J. Aguilar and K. Campero, studies the explicit speculative parallelism using a library of thread-level speculation, and presents the design of this library, which considers different speculative models: speculation of decision structures, speculation of loops, and speculation of critical sections. They evaluates different cases taken from SPEC CPU2000, allowing acceleration of about $1.8 \mathrm{x}$ in multicore architectures with coarse - grained multithreaded. The second one, entitled "Tuned Forest Fire Prediction: Static Calibration of the Evolutionary Component of 'ESS", by G. Bianchini and P. Caymes, presents an empirical study of the parameters tuning to evaluate the effectiveness of different configurations and the impact of the use of the Evolutionary-Statistical System (ESS) in the Forest Fires prediction. The ESS is a method whose aim is to reduce the uncertainty, by combining Statistical Analysis, High Performance Computing and Parallel Evolutionary Algorithms.

The Computer Graphics, Virtual Reality, and Image Processing Symposium has selected one paper. The paper "Accuracy and Efficiency Performance of the ICP Procedure Applied to Sign Language Recognition", by J. Silva, M. Lamar, and J. Bordim, addresses the problem of recognizing the American Sign Language (ASL), hand alphabet, relying only on depth information acquired from an RGB-D sensor. To accomplish this goal, a novel ICP-based recognition methodology is proposed where it analyzes the inputs and outputs of the alignment as efficiency and accuracy determinants. Next, a classification technique, denoted Approximated KBucket-fit, is proposed to efficiently handle the space complexity of the database template matching.

This selection shows the high level of the research being done in Latin America and presented at CLEI, we expect the reader to enjoy it.

We would like to thank all people involved in this undertaking, from the authors to the reviewers, for their effort and work, and CLEI steering committee and CLEI Electronic Journal for offering us the opportunity of preparing this special issue.
Jose Aguilar,
Jaelson Castro,
Sergio España,
Alexandra La Cruz, María Villapol,
Paula Zabala 